\title{
Evaluating Optic Nerve Damage: Pearls and Pitfalls
}

\author{
Paul J. Mackenzie* and Frederick S. Mikelberg
}

\begin{abstract}
Division of Glaucoma, Department of Ophthalmology and Visual Sciences, University of British Columbia, Vancouver Acute, 2550 Willow St, Vancouver, BC V5Z 3N9, Canada
\end{abstract}

\begin{abstract}
Primary open-angle glaucoma is a progressive optic neuropathy involving loss of retinal ganglion cells and their axons at the level of the optic nerve head. This change manifests as thinning and excavation of the neural tissues and nerve fiber layer. Therefore, it has long been known that the structural appearance of the optic nerve head is paramount to both glaucoma diagnosis and to the detection of progression [1-4]. Quantitative imaging methods such as Heidelberg Retinal Tomography (HRT) and Ocular Coherence Tomography (OCT) show great promise for the diagnosis and management of glaucoma and as these technologies continue to improve, they will become more important in the care of glaucoma. However, these tests cannot replace good clinical examination and indeed they depend upon clinical correlation for correct interpretation. Thus, careful and systematic clinical examination of the optic nerve remains a cornerstone of glaucoma management. In this paper, we outline a few pearls for the examination of the optic nerve and some of the pitfalls to be avoided in optic disc examination.
\end{abstract}

\section{OPTIC NERVE SIZE}

Any clinical assessment of the optic nerve needs to be made with respect to disc size. There is a large degree of variability in the size of the normal optic nerve, varying in area over a sevenfold ratio [5-7]. A large nerve can be expected to have a larger cup to disc ratio can be expected. Fig. (1) illustrates a large nerve with a physiologically large cup. Care must also be used when evaluation small optic nerves: even a small amount of cupping and rim thinning can represent glaucomatous damage.

\section{MEASUREMENT OF DISC SIZE}

Clinically, the optic disc is roughly equal to the small aperture (5 degrees) on the Welch Allan direct ophthalmoscope (or the medium spot size on a direct ophthalmoscope with three aperture sizes). Indirect slit-lamp biomicroscopy allows for measurement of the optic disc size using the vertical light scale on the slit lamp. The average vertical diameter of the optic disc around $1.8 \mathrm{~mm}$; a disc that is measured to be less than $1.5 \mathrm{~mm}$ is likely a small disc while one greater than $2.2 \mathrm{~mm}$ can be considered abnormally large. A correction factor must be used depending on the indirect lens that is used. For a 60 diopter lens, the correction factor is 1; that is, the measured size corresponds to the actual size. The correction factor is $1.1 \mathrm{x}$ and $1.3 \mathrm{x}$ respectively for a 78D and 90D lens.

Quantitative measurements of disc size can be obtained with imaging technologies. For example, the Heidelberg Retinal Tomograph (HRT) provides a measurement of disc size along with the determination of whether the optic disc is without or outside of the normal range. Fig. (2) shows HRT output for the optic nerve shown in Fig. (1); while Fig. (3)

*Address correspondence to this author at the Division of Glaucoma, Department of Ophthalmology and Visual Sciences, University of British Columbia, Vancouver Acute, 2550 Willow St, Vancouver, BC V5Z 3N9, Canada; E-mail: pmack@interchange.ubc.ca shows the normal standard automated perimetry (SAP) visual field from the same eye. The size of the optic disc is $4.00 \mathrm{~mm}^{2}$, significantly larger than the upper limit of normal of $2.43 \mathrm{~mm}^{2}$. Note as well that the Moorfield's Regression Analysis (MRA) suggests that this optic nerve has rim segments that are outside the normal range. Although this may represent early glaucoma, it may also be an example of the known decrease in specificity of the MRA with increasing disc size (and indeed all the statistical methods) $[8,9]$. This patient has remained stable with full visual fields off treatment and illustrates the potential pitfall of overreliance on technology or on one abnormal result. These optic nerve classifiers are meant are best used as part of the clinical context rather than as stand-alone decision-making tools.

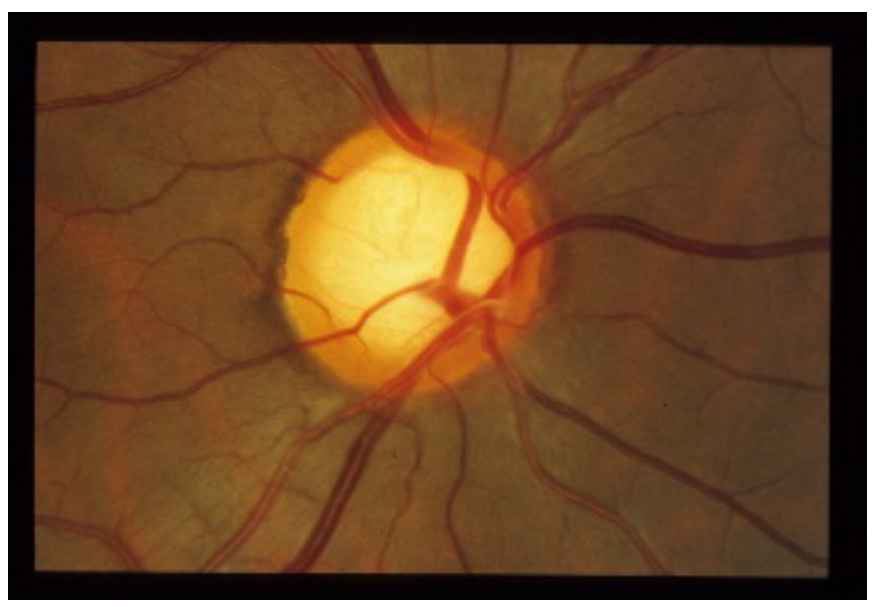

Fig. (1). Normal large optic nerve with corresponding physiologic cupping. Follows ISN'T rule.

\section{NEURORETINAL RIM}

The cup to disc ratio is a useful tool for assessing and communicating optic nerve health, but it is the remaining rim and not the cup that should be carefully examined. Focal 

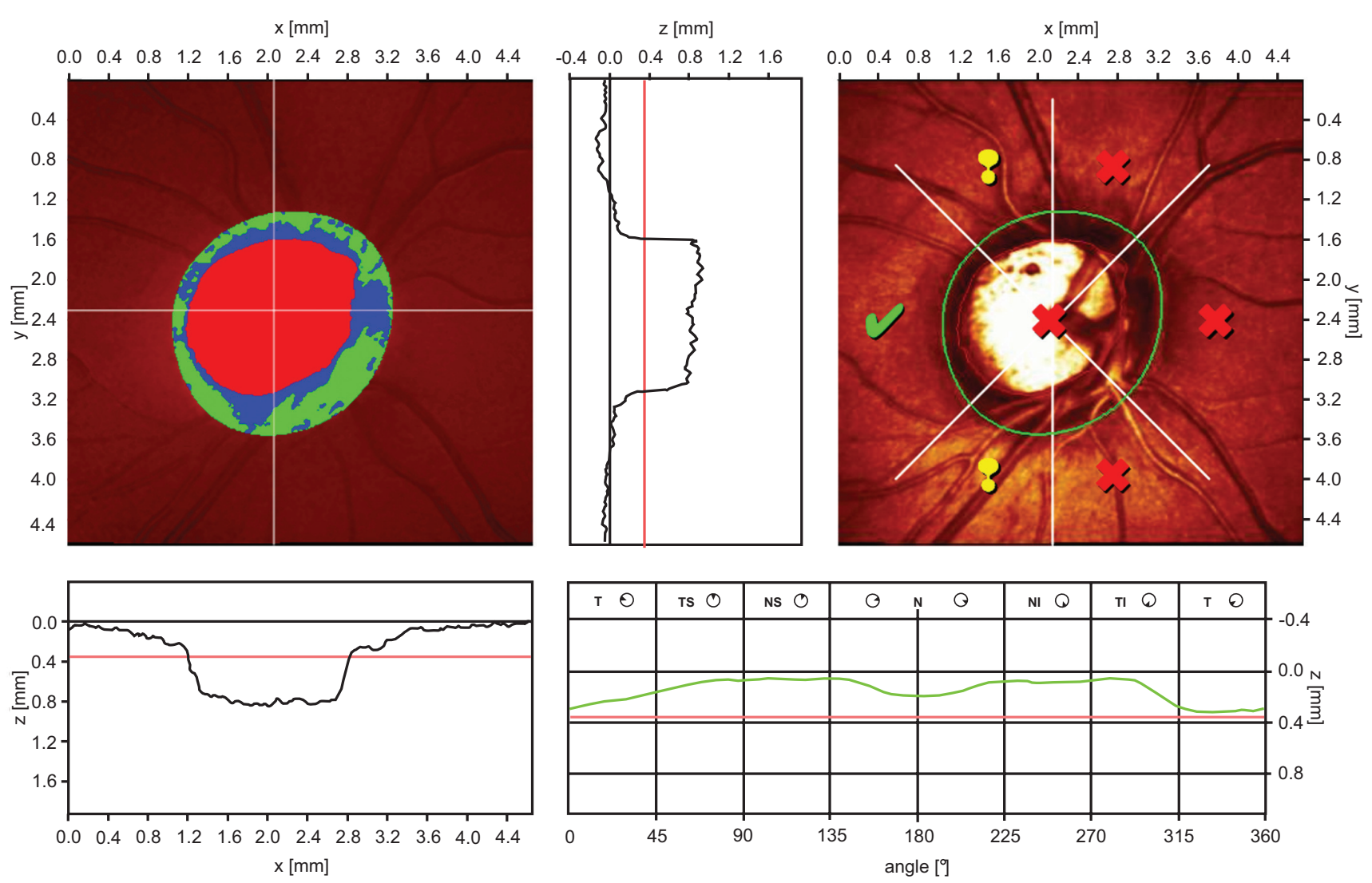

\begin{tabular}{|c|c|c|c|}
\hline Stereometric Analysis ONH & & Normal Range & 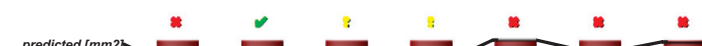 \\
\hline Disc Area & $3.91 \mathrm{~mm}^{2}$ & $1.63-2.43$ & \\
\hline Cup Area & $2.06 \mathrm{~mm}^{2}$ & $0.11-0.68$ & \\
\hline Rim Area & $1.85 \mathrm{~mm}^{2}$ & $1.31-1.96$ & \\
\hline Cup Volume & $0.80 \mathrm{~mm}_{3}$ & $-0.01-0.18$ & \\
\hline Rim Volume & $0.39 \mathrm{~mm}^{3}$ & $0.30-061$ & \multirow{3}{*}{ 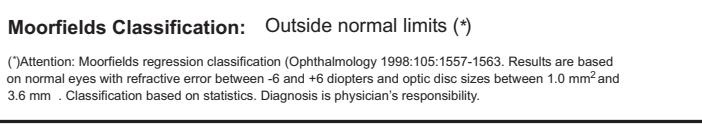 } \\
\hline Cup/Disc Area Ratio & 0.53 & $0.07-0.30$ & \\
\hline $\begin{array}{r}\text { Linear Cup/Disc Ratio } \\
\text { Mean Cup Depth }\end{array}$ & $\begin{array}{l}0.73 \\
0.44 \mathrm{~mm}\end{array}$ & $\begin{array}{l}0.27-0.55 \\
0.10-0.27\end{array}$ & \\
\hline $\begin{array}{l}\text { Maximum Cup Depth } \\
\text { Cup Shape Measure }\end{array}$ & $\begin{array}{l}0.81 \mathrm{~mm} \\
-0.02\end{array}$ & $\begin{array}{c}0.32-0.76 \\
-0.28--0.15\end{array}$ & \multirow[b]{8}{*}{ Date: 18/Jun/2009 Signature: } \\
\hline Height Variation Contour & $0.26 \mathrm{~mm}$ & $0.31-0.49$ & \\
\hline Mean RNFL Thickness & $0.21 \mathrm{~mm}$ & $0.20-0.32$ & \\
\hline RNFL Cross Sectional Area & $1.45 \mathrm{~mm}^{2}$ & $0.99-1.66$ & \\
\hline Reference Height & $355 \mu \mathrm{m}$ & & \\
\hline Topography Std Dev. & $9 \mu \mathrm{m}$ & & \\
\hline FSM & -0.46 & & \\
\hline$R B$ & 0.31 & & \\
\hline
\end{tabular}

Software Version: 3.1.2/949

Fig. (2). Heidelberg retinal tomography (HRT) output of the optic nerve shown in Fig. (1). See text for discussion of Moorfield's Regression Analysis abnormality in large optic nerve heads.

defects may be present and may indicate glaucoma, even in the presence of a "normal" cup to disc ratio. In order to avoid the pitfall of missing focal defects, the observer must pay attention to the entire circumference of the neuroretinal rim. The ISN'T rule is a useful mnemonic for recalling the expected relative thickness of the optic nerve segments. The
Inferior rim should be thickest, followed by the Superior, the Nasal and finally the Temporal rim. Fig. (4) illustrates a glaucomatous optic nerve that violates the ISN'T rule throughout the entire inferior rim, with further partial notching at 6 o'clock with an associated nerve fibre layer defect. 
Fixation Monitor: Gaze/Blind Spot Fixation Target: Central Fixation Losses: 0/16 False POS Errors: $1 \%$ False NEG Errors: $0 \%$ Test Duration: 05:28

Fovea: $38 \mathrm{~dB}$

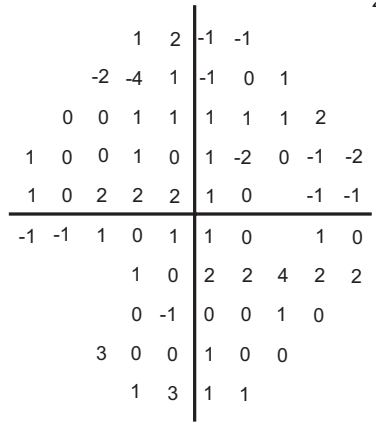

Total Deviation

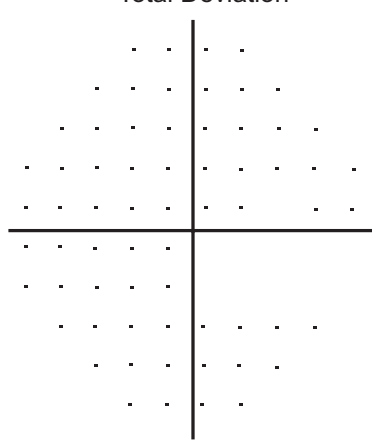

Stimulus: III, White Background: 31.5 ASB Strategy: SITA-Standard

Pupil Diameter: $8.4 \mathrm{~mm}$ Visual Acuity: RX: -0.75 DS $\quad$ DC X

Date: 05-01-2007 Time: 2:15 PM

Age: 54
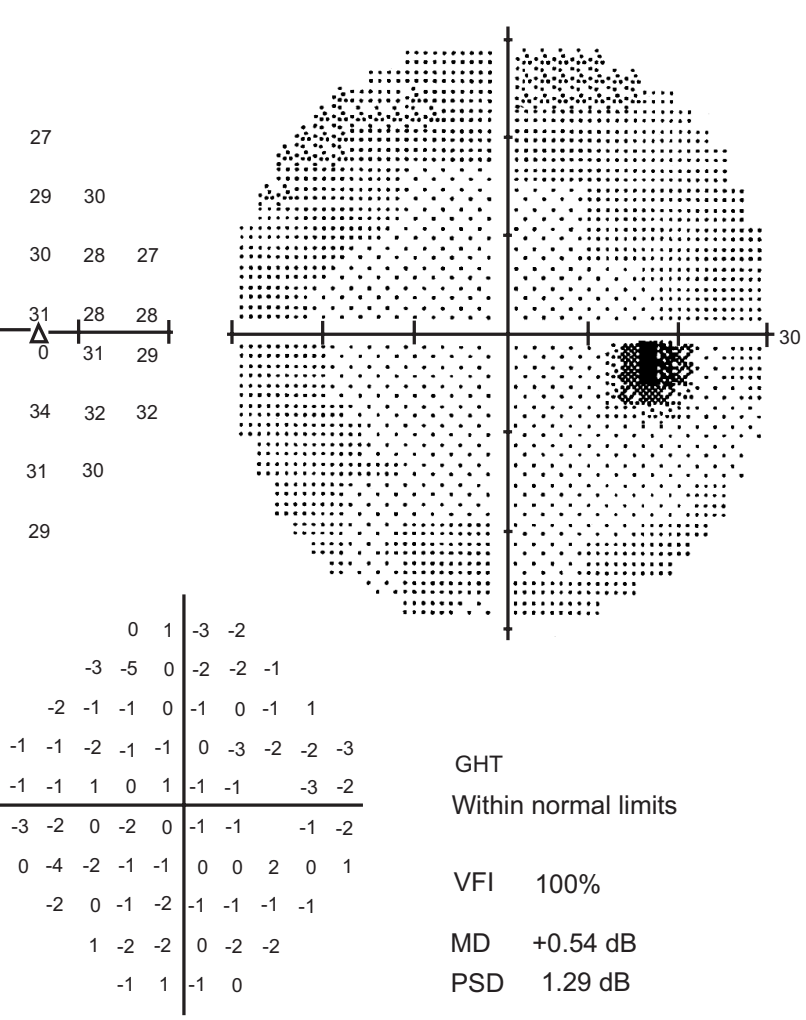

Pattern Deviation

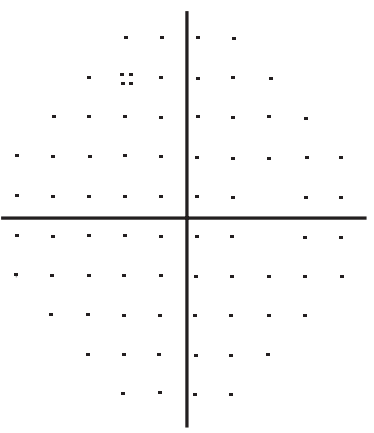

Vancover General Hospital Eye Care Centre 2550 Willow Street Vancouver, B.C SITA THREE

Fig. (3). White on white standard automated perimetry of optic nerve from Fig. (1) and HRT from Fig. (3). Normal visual field test. 


\section{RETINAL NERVE FIBRE LAYER EVALUATION}

The normal nerve fibre layer (NFL) can be visualized clinically, photographically or with optic nerve head analyzers such as ocular coherence tomography (OCT). The NFL can be detected clinically in most individuals, and is best appreciated using the red-free setting on the ophthalmoscope or slit-lamp. The NFL can be difficult to appreciate in the blond fundus. It appears as fine white striations radiating outwards from the optic nerve head and is normally most visible inferotemporally and superotemporally. It obscures details of the peripapillary retinal vasculature, particularly along the edges of vessels. A generalized thinning of the NFL can be best appreciated by comparison with the other half of the same fundus and with the other fundus; as well, generalized thinning can be detected by a greater prominence of the blood vessels, with sharper welldemarcated transition between vessel edge and retinal tissue. Localized NFL defects are easier to appreciate and can appear as the earliest sign of glaucomatous damage [10,11]. Although a NFL defect is not specific for glaucoma, it is pathological; therefore the presence of such a defect in an ocular hypertensive is fairly suggestive of glaucomatous damage [12].

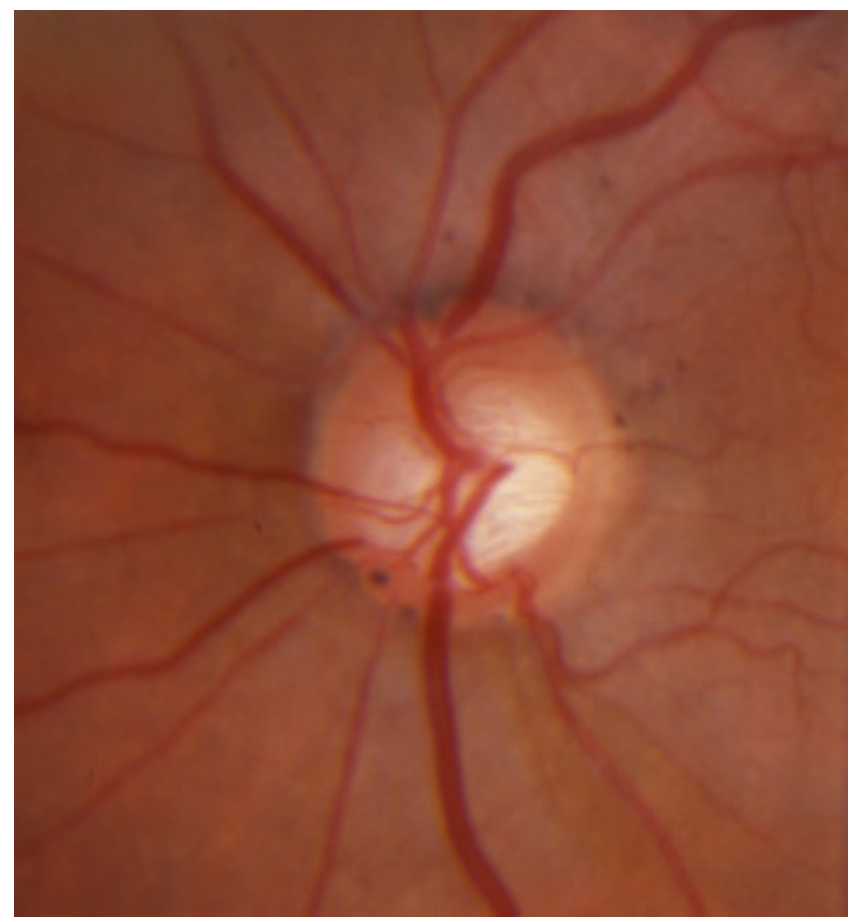

Fig. (4). Large optic nerve with glaucomatous cupping. Note violation of ISN'T rule inferiorly, along with inferior notch and associated nerve fibre layer wedge defect.

\section{DISC HEMORRHAGES}

It is important to look for splinter or flame-shaped hemorrhages at or around the optic nerve head as a sign of glaucomatous damage [13]. Fig. (5) illustrates a glaucomatous nerve with a disc hemorrhage with associated nerve fibre layer defect. On some occasions, disc hemorrhages have been shown to precede structural changes to the disc or nerve fibre layer by a period of weeks to months [14]. However, the Ocular Hypertensive Treatment Study found that although some disc hemorrhages predict progression, most (87\%) do not [15]. In addition, the Early Manifest Glaucoma Trial (EMGT) found that lowering IOP did not alter the rate of disc hemorrhage, suggesting that the presence of disc hemorrhage, rather than indicating insufficient IOP lowering, may indicate a less IOPdependent form of glaucoma [16]. Taken together, these results again support the theme that a single individual finding cannot be interpreted without reference to the overall clinical context. For example, the presence of a disc hemorrhage would justify treating a normotensive patient with a full visual field and healthy appearing optic nerve. On the other hand, in a patient with significant glaucoma who is being followed with borderline intraocular pressures, the appearance of a disc hemorrhage might tip the balance in favor of advancing therapy.

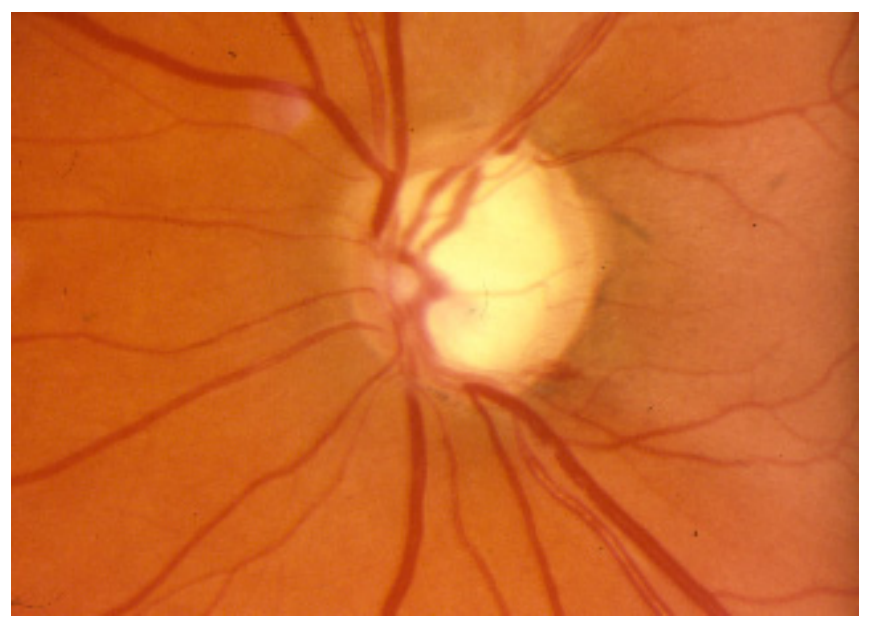

Fig. (5). Glaucomatous optic nerve with optic disc hemorrhage inferiorly, with associated nerve fibre layer defect.

\section{OPTIC NERVE PALLOR}

In most situations, there are clear differences between the clinical features of glaucoma and other optic neuropathies. However, on occasion, it is possible to be fooled into misdiagnosing non-glaucomatous insult, such as a compressive optic neuropathy, as glaucoma. Greenfield et al. [17]. have proposed the following criteria as suspicious for non-glaucomatous cupping in patients with pressures in the normal range: age less than 50 years, visual acuity less than 20/40, optic nerve pallor in excess of cupping, and vertically aligned visual field defects. However, Ahmed et al. [18] have reported that $6.5 \%$ of consecutive patients with what appeared to be clinically typical normal tension glaucoma were found to have a compressive optic neuropathy. They suggested that the Greenfield criteria, while specific for nonglaucomatous optic neuropathy may lack sensitivity. We apply these results in the following way. If a patient has one or more of the Greenfield criteria, they should undergo neuroimaging. However, the absence of these criteria does not rule out the presence of non-glaucomatous cupping and while we do not image everyone, we readily order neuroimaging if there is something unusual about the patient's glaucoma, including progression at very low apparent intraocular pressures.

\section{MYOPIA}

There are several potential pitfalls with respect to evaluating glaucoma in myopes, particularly high myopes. 
Myopic nerves are difficult to interpret, due to optic nerve tilt that makes evaluation of the temporal nerve, as well as the superior and inferior poles, difficult. However, usually some judgement can be made about the presence or absence of rim tissue at the superior and inferior poles and a nerve with no or little tissue present superiorly or inferiorly should not be disregarded as a myopic tilted nerve. Fig. (6) illustrates a glaucomatous nerve from a highly myopic patient. Although the inferior neuroretinal rim is somewhat obscured due to the tilt, the profile of the retinal vein as it courses inferiorly suggests excavation of rim tissue. An associated nerve fibre layer defect seen clinically and on OCT imaging, along with a corresponding superior arcuate defect on the visual field lend support to the diagnosis of glaucoma.

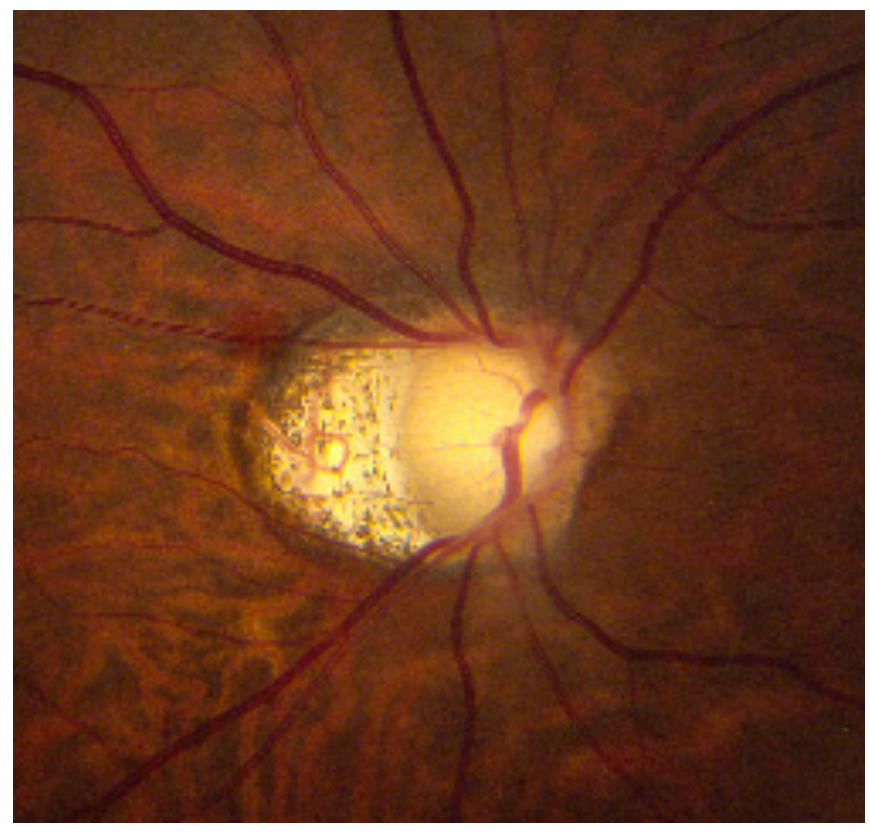

Fig. (6). Glaucomatous optic nerve in a highly myopic patient. See text for discussion of the clinical features.

Peripapillary atrophy can make determination of the optic disc margin difficult and can be misinterpreted as neuroretinal rim. In addition, glaucomatous cupping can be shallow and easily overlooked in a high myope. As well, the normalized databases for both visual fields and imaging technologies do not apply. Furthermore, other conditions such as myopic macular degeneration can complicate interpretation of visual fields. Finally, there are several arguments to suggest that highly myopic discs may be more susceptible to glaucomatous damage [19].

\section{SUMMARY}

Clinical evaluation of the optic nerve remains at the heart of glaucoma management, both for glaucoma diagnosis and for monitoring progression. Careful and systematic evaluation of the optic nerve is required in order to avoid potential pitfalls involving optic nerve size, focal rim defects, disc hemorrhages, and nerve fibre layer abnormalities. The reader is directed to the Jonas et al. major review of optic nerve evaluation for more detail [14]. Nonglaucomatous causes of optic neuropathy must always be considered. Finally, decisions should be based on the entire clinical picture and not on one isolated clinical or testing abnormality.

\section{REFERENCES}

[1] Drance SM. Correlation between optic disc changes and visual field defects in chronic open-angle glaucoma. Trans Am Acad Ophthalmol Otolaryngol 1976; 81: 224-6.

[2] Hoskins HD Jr., Gelber EC. Optic disk topography and visual field defects in patients with increased intraocular pressure. Am J Ophthalmol 1975; 80: 284-90.

[3] Hitchings RA, Spaeth GL. The optic disc in glaucoma II: Correlation of the appearance of the optic disc with the visual field. Br J Ophthalmol 1977; 61: 107-13.

[4] Susanna R, Drance SM. Use of discriminant analysis I. Prediction of visual field defects from features of the glaucoma disc. Arch Ophthalmol 1978; 96: 1568-70.

[5] Britton RJ, Drance SM, Schulzer MD, et al. The area of the neuroretinal rim of the optic nerve in normal eyes. Am J Ophthalmol 1987; 103: 497-504.

[6] Jonas JB, Gusek GC, Naumann GOH. Optic disk, cup and neuroretinal rim size, configuration, and correlations in normal eyes. Invest Ophthalmol Vis Sci 1988; 29: 1151-8.

[7] Varma R, Tielsch JM, Quigley HA, et al. Race-, age-, gender-, and refractive error-related differences in the normal optic disk. Arch Ophthalmol 1994; 112: 1068-76.

[8] Ford BA, Artes PH, McCormick TA, et al. Comparison of data analysis tools for detection of glaucoma with the Heidelberg Retina Tomograph. Ophthalmology 2003; 110: 1145-50.

[9] Yip LW, Mikelberg FS. A comparison of the glaucoma probability score to earlier heidelberg retina tomograph data analysis tools in classifying normal and glaucoma patients. J Glaucoma 2008; 17 : 513-6.

[10] Airaksinen PJ, Mustonen E, Alanku HI. Optic disk haemorrhages precede retinal nerve fibre layer defects in ocular hypertension. Acta Ophthalmol 1981; 59: 627-41.

[11] Sommer A, Katz J, Quigley HA, et al. Clinically detectable nerve fiber atrophy precedes the onset of glaucomatous field loss. Arch Ophthalmol 1991; 109: 77-83.

[12] Jonas JB, Budde WM, Panda-Jonas S. Ophthalmoscopic evaluation of the optic nerve head. Surv Ophthalmol 1999; 43: 293-320.

[13] Drance SM, Begg IS. Sector hemorrhage: a probable acute disk change in chronic simple glaucoma. Can J Ophthalmol 1970; 5: 137-41.

[14] Heijl A. Frequent disk photography and computerized perimetry in eyes with optic disk haemorrhage. Acta Ophthalmol 1986; 64: 27481 .

[15] Budenz DL, Anderson DR, Feuer WJ. Detection and prognostic significance of optic disc hemorrhages during the ocular hypertension treatment study. Ophthalmology 2006; 113: 2137-43.

[16] Bengtsson B, Leske MC, Yang Z, Heijl A, EMGT group. disc hemorrhages and treatment in the early manifest glaucoma trial. Ophthalmology 2008; 115: 2044-8.

[17] Greenfield DS, Siatkowski RM, Glaser JS, et al. The cupped disc: who needs neuroimaging? Ophthalmology 1998; 105: 1866-74.

[18] Ahmed IIK, Feldman F, Kucharczyk W, Trope GE. Neuroradiologic screening in normal-pressure glaucoma: study results and literature review. J Glaucoma 2002; 11:279-86.

[19] Burgoyne CF. Myopic eyes and glaucoma. J Glaucoma 2004; 13 : 85-6. 